\title{
Consumo de bebidas alcoólicas e obesidade abdominal: resultados da linha de base do ELSA-Brasil
}

\author{
Consumption of alcoholic beverages and abdominal obesity: \\ cross-sectional analysis of ELSA-Brasil
}

Gabrielle Guidoni Torres (https://orcid.org/0000-0003-3091-4076) ${ }^{1}$ Jordana Herzog Siqueira (https://orcid.org/0000-0003-0116-7411) ${ }^{1}$ Oscar Geovanny Enriquez Martinez (https://orcid.org/0000-0003-4561-122X) ${ }^{1}$ Taísa Sabrina Silva Pereira (https://orcid.org/0000-0002-5922-7424) ${ }^{2}$ Jorge Gustavo Velásquez Meléndez (https://orcid.org/0000-0001-8349-5042) ${ }^{3}$ Bruce Bartholow Duncan (https://orcid.org/0000-0002-7491-2630) ${ }^{4}$ Alessandra Carvalho Goulart (https://orcid.org/0000-0003-1076-5210) ${ }^{5}$ Maria del Carmen Bisi Molina (https://orcid.org/0000-0001-8746-5860) ${ }^{6}$

${ }^{1}$ Programa de PósGraduação em Saúde Coletiva, Universidade Federal do Espírito Santo. Av. Marechal Campos, 1.468, Bonfim. 29047105 Vitória ES Brasil. gabrielleguidoni@ gmail.com

${ }^{2}$ Nutrition Science, Department of Health Sciences, University of the Americas Puebla. Puebla México.

${ }^{3}$ Departamento de Enfermagem MaternoInfantil e Saúde Pública, Escola de Enfermagem. Belo Horizonte MG Brasil.

${ }^{4}$ Departamento de Medicina Social, Faculdade de Medicina, Universidade Federal do Rio Grande do Sul. Porto Alegre RS Brasil. ${ }^{5}$ Centro de Pesquisas Clínicas e Epidemiológicas, Hospital Universitário, Universidade de São Paulo. São Paulo SP Brasil. ${ }^{6}$ Programa de PósGraduação em Saúde e Nutrição, Universidade Federal de Ouro Preto. Ouro Preto MG Brasil.

\begin{abstract}
The objective was to analyze the association between alcohol consumption and abdominal adiposity in adults. Cross-sectional study conducted at baseline data from ELSA-Brasil (2008-2010). The sample consisted of 15,065 civil servants from six education and research institutions (35 to 74 years old, both sexes). To identify central adiposity by measuring waist circumference (WC) and waist-to-hip ratio (WHR), the cutoff points recommended by the World Health Organization were used. Poisson regression models adjusted for potentially confounding variables were tested. About $40 \%$ of the sample had elevated WC and WHR. The probability of having elevated WC was 5\% and 3\% higher in the most exposed group of beer consumption in men and women when compared to the reference group $[P R=1.05$ (95\% CI 1.02-1.08) and $P R=$ 1.03 (95\% CI 1.00-1.07)]. A higher probability of having a high WHR was also found among the highest beer consumers $[P R=1.03(95 \% C I$ 1.00-1.07) in men and $P R=1.10$ (95\% CI 1.041.15) in women]. A greater number of doses/ week of alcoholic drink increased the probability of occurrence of high WC and WHR, with the beer contribution being more important.
\end{abstract}

Key words Obesity, Abdominal obesity, Alcohol drinking, Waist circumference, Waist-to-hip ratio
Resumo Objetivou-se analisar a associação entre consumo de bebidas alcoólicas e adiposidade abdominal em adultos. Estudo transversal realizado com dados da linha de base do ELSA-Brasil (2008-2010). A amostra foi constituída por 15.065 servidores públicos de seis instituições de ensino e pesquisa (35 a 74 anos, ambos os sexos). Para identificar adiposidade central por meio das medidas de circunferência da cintura (CC) e relação cintura/quadril (RCQ), utilizou-se os pontos de corte preconizados pela Organização Mundial da Saúde. Para as análises estatísticas foi utilizado o teste qui-quadrado e modelos de regressão de Poisson ajustados por variáveis potencialmente confundidoras. Cerca de $40 \%$ da amostra apresentava CC e RCQ elevadas. A probabilidade de apresentar CC elevada foi $5 \%$ e $3 \%$ maior no grupo mais exposto de consumo de cerveja em homens e mulheres quando comparado ao grupo de referência $[R P=1,05$ (IC 95\% 1,02-1,08) e $R P=1,03$ (IC 95\% 1,00-1,07)]. Também foi encontrada maior probabilidade de apresentar RCQ elevada entre os maiores consumidores de cerveja $[R P=1,03$ (IC 95\% 1,00-1,07) em homens e $R P=1,10$ (IC 95\% 1,04-1,15) em mulheres]. Maior número de doses/semana de bebida alcoólica aumentou a probabilidade de ocorrência de CC e RCQ elevadas, sendo mais importante a contribuição da cerveja. Palavras-chave Obesidade, Obesidade abdominal, Consumo de bebidas alcoólicas, Circunferência da cintura, Relação cintura-quadril 


\section{Introdução}

A obesidade é um importante problema de saúde pública e vem ganhando destaque no cenário epidemiológico mundial. Sua prevalência aumentou significativamente em todo o mundo nas últimas décadas ${ }^{1}$, e acredita-se que os determinantes do excesso de peso compõem um complexo conjunto de fatores biológicos, comportamentais, ambientais, sociais e econômicos. Além de ser considerada uma doença, a obesidade é também um importante fator de risco para o desenvolvimento de inúmeras condições adversas à saúde ${ }^{2}$, visto que a gordura abdominal tem grande contribuição na determinação do risco cardiovascular ${ }^{3,4} \mathrm{e}$ de alterações metabólicas ${ }^{5,6}$. Nesse sentido, a circunferência da cintura (CC) parece explicar melhor o risco à saúde relacionado à obesidade do que o índice de massa corporal (IMC) ${ }^{7}$.

Vários fatores estão associados à obesidade abdominal, como idade, sexo, raça/cor, condições socioeconômicas e variáveis comportamentais relacionadas ao estilo de vida ${ }^{8,9}$, entre as quais o consumo de bebidas alcoólicas ${ }^{10,11}$. Apesar de esse consumo representar um hábito comum e milenar em muitas sociedades ${ }^{12}$, meta-análises recentes ${ }^{13,14}$ evidenciaram que o uso de álcool, independentemente da quantidade, representa risco à saúde.

No mundo, cerca de 2 bilhões de pessoas consomem bebidas alcoólicas por ano, o que corresponde a aproximadamente $40 \%$ da população mundial acima de 15 anos $^{12}$. No Brasil, o consumo anual de álcool puro per capita foi estimado em 8,7 litros por adulto, quantidade acima da média mundial $(6,2 \text { litros })^{12}$.

O álcool é obtido por meio da fermentação de carboidratos presentes em alguns alimentos e fornece $7,1 \mathrm{kcal} / \mathrm{g}$ para o organismo, embora seja uma fonte de energia diferente devido à sua toxicidade. Há evidências que o consumo excessivo de bebidas alcoólicas pode produzir aumento de depósitos de gordura na região abdominal via metabolismo do álcool ${ }^{15}$.

Como o álcool não pode ser armazenado no organismo, rapidamente é metabolizado, alterando outras vias metabólicas, incluindo a oxidação lipídica, favorecendo o estoque de gorduras no organismo, com deposição preferencialmente na área abdominal ${ }^{16}$. Vários estudos transversais avaliaram a associação entre consumo de bebidas alcoólicas e obesidade abdominal, mas os resultados ainda são inconclusivos, sobretudo pela grande variação metodológica encontrada na literatura relativa à análise da frequência e da quantidade de etanol consumido, além das variáveis utilizadas nos ajustes dos modelos estatísticos. Apesar de estudos terem encontrado associação nula ou negativa entre consumo de álcool e obesidade abdominal ${ }^{17-19}$, outros evidenciaram que o consumo de cerveja se associa positivamente à obesidade abdominal e que o vinho pode ser um fator protetor ${ }^{11,20,21}$.

Diante desse cenário, o objetivo do presente estudo foi analisar a associação entre consumo de bebidas alcoólicas e adiposidade abdominal em adultos brasileiros.

\section{Métodos}

\section{Desenho e população do estudo}

Trata-se de um estudo transversal realizado com dados da linha de base (2008-2010) do Estudo Longitudinal de Saúde do Adulto (ELSA-Brasil), com 15.105 servidores públicos, ativos e aposentados, com idade entre 35 e 74 anos, de ambos os sexos, de seis instituições de ensino superior localizadas em seis estados brasileiros (São Paulo, Minas Gerais, Bahia, Rio Grande do Sul, Rio de Janeiro e Espírito Santo). O principal objetivo do ELSA-Brasil foi investigar a incidência e os determinantes de doenças crônicas não transmissíveis na população brasileira, com foco nas doenças cardiovasculares e diabetes. As características gerais da coorte foram publicadas anteriormente ${ }^{22}$.

Os participantes, em um dia previamente agendado, compareceram ao centro de investigação (CI) no turno matutino para realização de exames clínicos, bioquímicos e entrevista. Informações gerais sobre aferição e exames clínicos podem ser encontradas em publicação anterior ${ }^{23}$. Foram considerados para análise os participantes que responderam ao questionário de avaliação do consumo de bebidas alcoólicas e com dados antropométricos e sociodemográfícos completos na linha de base. Assim, foram excluídos os participantes que não tinham dados de IMC $(\mathrm{n}=26)$, CC $(n=12)$ e circunferência do quadril (CQ) (n $=2$ ). A amostra final consistiu em 15.065 participantes.

\section{Variáveis de interesse}

\section{Dados antropométricos}

As medidas corporais (peso, altura, CC e CQ) foram aferidas de acordo com procedimentos padronizados ${ }^{24}$. Todas as medidas antropométricas foram feitas com os participantes em jejum e 
com a bexiga vazia. O peso corporal foi aferido com o participante descalço, trajando um uniforme padrão sobre as roupas íntimas. Utilizouse balança eletrônica (Toledo, modelo 2096PP), com capacidade de $200 \mathrm{~kg}$ e precisão de $50 \mathrm{~g}$. A altura foi aferida com estadiômetro de parede (Seca, Hamburg, BRD) com precisão de $1 \mathrm{~mm}$, afixado à parede lisa e sem rodapé. $\mathrm{O}$ indivíduo estava descalço, encostando cabeça, nádegas e calcanhares na parede e com o olhar fixo no plano horizontal. A estatura foi verificada no período inspiratório do ciclo respiratório. A partir dos dados de peso e altura, foi calculado o IMC (peso corporal dividido pela altura ao quadrado - $\mathrm{kg} /$ $\mathrm{m}^{2}$ ) e utilizados os critérios da OMS para classificação do estado nutricional.

A CC foi aferida com o participante em posição ereta, respirando normalmente, com os pés juntos, a parte superior da vestimenta (blusa) erguida e com os braços cruzados na frente do peito. A medida foi feita com uma fita métrica inextensível no ponto médio entre a crista ilíaca e a borda inferior do último arco costal. A CC elevada foi identificada quando maior ou igual a 94 $\mathrm{cm}$ e $80 \mathrm{~cm}$, respectivamente, para homens e mulheres. A CQ foi aferida na maior proeminência das nádegas e a partir dessas medidas foi calculada a relação cintura-quadril (RCQ). A RCQ elevada foi identificada quando $\geq 1$ e $\geq 0,85$, respectivamente, para homens e mulheres. As variáveis CC e RCQ foram categorizadas de acordo com os pontos de corte recomendados pela Organização Mundial da Saúde ${ }^{25}$, definindo-se assim a CC e RCQ elevadas, que foram as variáveis dependentes do estudo.

\section{Consumo de álcool}

O consumo de bebidas alcoólicas foi relatado por meio de questionários estruturados com perguntas fechadas, realizados em cada CI do ELSA-Brasil, para determinar os tipos de bebidas alcoólicas (cerveja, vinho e destiladas - cachaça, vodka e whisky). Também foram determinadas a frequência (diário, semanal, mensal) e a quantidade de consumo em ml/dia ${ }^{26}$. A classificação de uma dose foi estabelecida para as diferentes bebidas alcoólicas, considerando: uma taça de vinho tinto ou branco $(120 \mathrm{ml})$, uma lata/long neck de cerveja $(350 \mathrm{ml})$ ou uma garrafa de cerveja de 620 $\mathrm{ml}$ (duas doses). Para as bebidas destiladas, foram consideradas $50 \mathrm{ml}$ para uma dose de cachaça, vodka ou whisky.

Sendo assim, cada tipo de bebida (cerveja, vinho e destiladas) e todas as bebidas alcoólicas (somatório) foram classificadas em dose/semana e, posteriormente, esse consumo em dose/semana foi dividido em quintis para todas as análises.

\section{Covariáveis}

\section{Sociodemográficas}

As variáveis sociodemográficas foram coletadas por meio de questionário padronizado em entrevista realizada em cada CI do ELSA-Brasil. A idade foi categorizada em quatro grupos (3544 anos, 45-54 anos, 55-64 anos e 65-74 anos). A raça/cor da pele foi autorreferida, categorizada em branca e não/branca (parda, preta, amarela e indígena). A escolaridade foi categorizada como fundamental incompleto, fundamental completo, médio e superior/pós-graduação. A renda per capita familiar foi calculada com base no valor líquido total de rendimento da família em reais nos últimos três meses, e dividido pela quantidade de pessoas que dependem da renda para viver e posteriormente subdividida em tercis.

\section{Variáveis do estilo de vida}

Em relação ao tabagismo, a variável foi categorizada em "ex-fumante", "fumante atual" e "nunca fumou". Para medir o nível de atividade física no lazer, foi utilizado o Questionário Internacional de Atividade Física (IPAQ) versão longa, validado para o Brasil ${ }^{27}$, nos domínios atividade física de lazer em tempo livre e atividade física de deslocamento. A variável atividade física foi registrada em minutos/semana e subdividida em fraca, moderada e forte.

\section{Análise estatística}

O teste qui-quadrado foi utilizado para avaliar a diferença de proporções. A associação entre variáveis dependentes (CC e RCQ elevadas) e os quintis de consumo de bebidas alcoólicas (variável independente) foi analisada a partir de modelos de Poisson (RP e IC 95\%), ajustados por idade, renda per capita, IMC, tabagismo e atividade física.

O critério utilizado para a seleção das variáveis de ajuste no modelo foram a plausibilidade biológica e teórica avaliada pelo DAG, não incluindo as variáveis colisoras e aplicando o modelo mínimo de ajuste e o critério estatístico ( $\mathrm{p} \leq$ 0,20 na análise bivariada). Como escolaridade e renda per capita são colineares, foi escolhida apenas uma.

O nível de significância para todos os testes foi de $\mathrm{p}<0,05$. Os dados foram analisados por meio do programa estatístico Statistical Package for the Social Sciences - SPSS 17.0. 


\section{Resultados}

Foram avaliados 15.065 participantes (45,6\% homens e $54,4 \%$ mulheres), com idade média de 52 anos (DP $\pm 9,0$ ), e cerca de $40 \%$ da amostra apresentava CC e RCQ elevadas. O consumo de bebidas alcoólicas foi observado em quase metade dos indivíduos estudados, sendo a cerveja a bebida mais consumida em ambos os sexos, seguida pelas bebidas destiladas em homens e o vinho entre as mulheres. Os homens consumiram em média o dobro de cerveja e bebidas destiladas em doses/semana quando comparados às mulheres. O consumo médio de vinho em doses/semana também diferiu entre os homens (3,6 doses/ semana em homens versus 2,8 doses/semana em mulheres) (dados não apresentados em tabelas). Quando analisada a distribuição da amostra segundo a classificação da CC e RCQ, observou-se significância estatística para todas as variáveis sociodemográficas e de estilo de vida analisadas (Tabela 1).

Maiores prevalências de CC foram observadas em indivíduos com idade entre 45 e 54 anos, que relataram praticar atividade física fraca, possuem nível de escolaridade alta (superior/pós-graduação) e que relataram não ser fumantes, em ambos os sexos. Nos homens, a CC elevada se associa ao sobrepeso, raça/cor branca e último tercil de renda per capita, enquanto em mulheres foi observada associação com obesidade, raça/cor não branca e tercil de renda per capita mais baixo. Maiores prevalências de RCQ foram encontradas em participantes que praticavam atividade física fraca, em ambos os sexos. Nos homens, a RCQ elevada se associou a idade (55 a 64 anos), obesidade, ensino médio completo, raça/cor branca, ex-fumantes e maior tercil de renda per capita. Nas mulheres, associou-se a idade (45 a 54 anos), sobrepeso, escolaridade superior/pós-graduação, raça/cor não branca, não fumantes, e no mais baixo tercil de renda per capita (Tabela 1).

Entre os homens, os quintis de consumo (dose/semana) de vinho ( $\mathrm{p}=0,002)$, cerveja $(\mathrm{p}<$ $0,001)$ e todas as bebidas alcoólicas analisadas ( $\mathrm{p}$ $>0,005)$ foram associadas à CC elevada. Já entre as mulheres, a associação foi encontrada apenas para a cerveja $(\mathrm{p}<0,001)$ e todas as bebidas alcoólicas analisadas $(\mathrm{p}=0,004)$. Em relação à $\mathrm{RCQ}$, entre os homens os quintis de consumo (dose/ semana) de todas as bebidas alcoólicas analisadas $(\mathrm{p}<0,001)$, vinho $(\mathrm{p}=0,004)$ e cerveja $(\mathrm{p}<$ $0,001)$, e entre as mulheres cerveja $(\mathrm{p}<0,001)$ e todas as bebidas alcoólicas analisadas $(\mathrm{p}<0,001)$ se associaram à RCQ elevada (Tabela 2).
Após ajuste por variáveis condundidoras, a probabilidade de apresentar CC elevada foi $5 \%$ e 3\% maior no grupo mais exposto de consumo de cerveja (quinto quintil de doses/semana) em homens e mulheres, quando comparado ao grupo de referência $[\mathrm{RP}=1,05$ (IC 95\% 1,02-1,08) e $\mathrm{RP}=1,03$ (IC 95\% 1,00-1,07), respectivamente]. Também foi encontrada maior probabilidade de apresentar RCQ elevada entre os maiores consumidores de cerveja (quinto quintil de doses/ semana) [ $\mathrm{RP}=1,03$ (IC 95\% 1,00-1,07) em homens e RP $=1,10$ (IC 95\% 1,04-1,15) em mulheres]. Não foi encontrada associação entre o consumo de vinho e bebidas destiladas com CC e RCQ elevadas (tabelas 3 e 4).

\section{Discussão}

Cerca de $40 \%$ da amostra apresentou CC e RCQ elevadas, e quase metade referiu consumir bebidas alcoólicas, sendo a cerveja a bebida mais consumida em ambos os sexos, seguida pelas bebidas destiladas em homens e vinho entre as mulheres. O consumo de cerveja se associou a CC e RCQ elevadas em homens e mulheres após ajuste por variáveis de confusão.

O consumo de cerveja mostrou-se associado a CC e RCQ, tanto em homens quanto em mulheres. No Brasil, Ferreira e colaboradores, em estudo transversal com 1.749 homens doadores de sangue evidenciou que a prevalência de inadequação tanto da CC quanto da RCQ aumentou linearmente com a quantidade de álcool ingerida ${ }^{28}$. Estudo realizado com dados da Pesquisa Nutrição e Saúde (PNS) identificou que a prevalência de RCQ elevada entre os homens se associou à maior quantidade diária de cerveja, e não houve associação com o consumo de bebidas destiladas. A prevalência de RCQ elevada aumentou com a idade em ambos os sexos e de forma mais acentuada em mulheres acima dos 50 $\operatorname{anos}^{29}$.

Uma revisão sistemática abordou a relação entre o consumo de cerveja e obesidade abdominal, por meio da avaliação de CC e RCQ, em estudos transversais e experimentais. Os achados evidenciaram associações positivas em homens, mas dados inconsistentes em mulheres ${ }^{30}$. Esses resultados foram explicados pela diferença de consumo de bebidas alcoólicas entre os sexos, observando maior consumo entre os homens ${ }^{31}$.

Nesse cenário, a maior parte dos trabalhos relacionados ao consumo de bebidas alcoólicas tratam separadamente homens e mulheres, por suas 
Tabela 1. Distribuição da amostra segundo circunferência da cintura e relação cintura-quadril (ELSA - Brasil, 2008-2010).

\begin{tabular}{|c|c|c|c|c|c|c|c|c|c|}
\hline \multirow{4}{*}{ Variáveis } & \multicolumn{5}{|c|}{ Circunferência da cintura } & \multicolumn{4}{|c|}{ Relação cintura-quadril } \\
\hline & \multirow[b]{2}{*}{ Total } & \multicolumn{2}{|c|}{ Masculino } & \multicolumn{2}{|c|}{ Feminino } & \multicolumn{2}{|c|}{ Masculino } & \multicolumn{2}{|c|}{ Feminino } \\
\hline & & Elevada & P-valor & Elevada & P-valor & Elevada & P-valor & Elevada & P-valor \\
\hline & n (\%) & n (\%) & & n (\%) & & n (\%) & & n (\%) & \\
\hline Idade (anos) & & & $<0,001$ & & $<0,001$ & & $<0,001$ & & $<0,001$ \\
\hline 35 a 44 & $3334(22,1)$ & $651(17,9)$ & & $348(15,1)$ & & $176(10,3)$ & & $595(14,2)$ & \\
\hline 45 a 54 & $5926(39,3)$ & $1424(39,2)$ & & $868(37,7)$ & & $562(32,9)$ & & $1560(37,2)$ & \\
\hline 55 a 64 & $4221(28)$ & $1080(29,8)$ & & $762(33,1)$ & & $625(36,6)$ & & $1453(34,7)$ & \\
\hline 65 a 74 & $1584(10,5)$ & $474(13.1)$ & & $323(14,0)$ & & $344(20,2)$ & & $583(13,9)$ & \\
\hline Estado nutricional & & & $<0,001$ & & $<0,001$ & & $<0,001$ & & $<0,001$ \\
\hline Magreza & $3334(0,9)$ & - & & - & & - & & & \\
\hline Eutrofia & $5926(35,9)$ & $182(5,0)$ & & $11(0,5)$ & & $139(8,1)$ & & $868(20,7)$ & \\
\hline Sobrepeso & $4221(40,3)$ & $2045(56,4)$ & & $559(24,3)$ & & $139(8,1)$ & & $1698(40,5)$ & \\
\hline Obesidade & $1584(22,9)$ & $1401(38,6)$ & & $1730(75,2)$ & & $800(46,9)$ & & $1617(38,6)$ & \\
\hline Atividade física & & & $<0,001$ & & $<0,001$ & & $<0,001$ & & $<0,001$ \\
\hline Fraca & $11430(77)$ & $2763(77,2)$ & & $1939(85,2)$ & & $1345(79,8)$ & & $3425(82,8)$ & \\
\hline Moderada & $2072(14)$ & $514(14,3)$ & & $247(10,8)$ & & $234(13,9)$ & & $496(12,0)$ & \\
\hline Forte & $1347(9)$ & $304(8,5)$ & & $91(4,0)$ & & $106(6,3)$ & & $213(5,2)$ & \\
\hline Escolaridade & & & 0,005 & & $<0,001$ & & $<0,001$ & & $<0,001$ \\
\hline Fundamental incompleto & $888(5,9)$ & $296(8,2)$ & & $153(6,6)$ & & $197(1(1,5)$ & & $252(6,0)$ & \\
\hline Fundamental completo & $1025(6,8)$ & $294(8,1)$ & & $183(8,0)$ & & $168(9,8)$ & & $310(7,4)$ & \\
\hline Médio completo & $5218(34,6)$ & $1136(31,3)$ & & $930(40,4)$ & & $488(28,6)$ & & $1621(38,7)$ & \\
\hline Superior/pós-graduação & $7934(52,7)$ & $1903(52,4)$ & & $1035(45,0)$ & & $854(50)$ & & $2008(47,9)$ & \\
\hline Raça/cor & & & $<0,001$ & & $<0,001$ & & $<0,001$ & & $<0,001$ \\
\hline Não Branca & $7118(47,77)$ & $1578(44,1)$ & & $1216(53,4)$ & & $690(41,0)$ & & $2161(52,0)$ & \\
\hline Branca & $7780(52,23)$ & $2003(55,9)$ & & $1061(46,6)$ & & $991(59,0)$ & & $1994(48,0)$ & \\
\hline Tabagismo & & & $<0,001$ & & $<0,001$ & & $<0,001$ & & $<0,001$ \\
\hline Nunca fumou & $8571(56,9)$ & $1665(45,9)$ & & $1356(58,9)$ & & $688(40,3)$ & & $2461(58,7)$ & \\
\hline Ex-fumante & $4522(30)$ & $1507(41,5)$ & & $676(29,4)$ & & $777(45,5)$ & & $1187(28,3)$ & \\
\hline Fumante & $1971(13,1)$ & $457(12,6)$ & & $269(11,7)$ & & $241(14,1)$ & & $543(13,0)$ & \\
\hline Renda per capita & & & 0,001 & & $<0,001$ & & 0,001 & & $<0,001$ \\
\hline $1^{\circ}$ tercil & $4955(33)$ & $1196(33,1)$ & & $866(37,8)$ & & $540(31,7)$ & & $1474(35,3)$ & \\
\hline $2^{\circ}$ tercil & $4792(31,9)$ & $1118(30,9)$ & & $721(31,4)$ & & $544(32,0)$ & & $1365(32,7)$ & \\
\hline $3^{\circ}$ tercil & $5264(35,1)$ & $1303(36,0)$ & & $705(30,8)$ & & $618(36,3)$ & & $1340(32,1)$ & \\
\hline
\end{tabular}

${ }^{*}$ Teste qui-quadrado $(\mathrm{p}<0,05)$.

Fonte: Autores.

diferenças de metabolismo e consumo ${ }^{32}$, sendo que maior proporção de homens são bebedores excessivos (cinco doses ou mais em uma ocasião nos últimos 12 meses) $)^{33}$. Em estudos epidemiológicos, a associação entre o consumo de bebidas alcoólicas e peso corporal varia de acordo com o sexo. Assim, o metabolismo de primeira passagem gástrica é reduzido nas mulheres e o volume de distribuição de uma dose de álcool é inferior em mulheres. Devido à maior massa de gordura corporal, as mulheres obtêm uma concentração de álcool no sangue maior do que os homens após a ingestão de uma dose definida de álcool ${ }^{15}$.
A prevalência de excesso de peso e obesidade nesta amostra foi maior do que a encontrada na população brasileira em geral ${ }^{34}$. Diferentemente do presente estudo, pesquisa realizada por Pinho e colaboradores com indivíduos na faixa etária de 25 a 59 anos do estado de Pernambuco identificou maior prevalência de obesidade abdominal no sexo feminino, sobretudo a partir da terceira década de vida, e na menor faixa de escolaridade, além de maior consumo de bebidas alcoólicas em homens, quando comparados com mulheres ${ }^{8}$.

Apesar de outras pesquisas demonstrarem que o consumo leve e moderado de álcool repre- 
Tabela 2. Consumo de bebidas alcoólicas (doses/semana), segundo circunferência da cintura e relação cinturaquadril (ELSA - Brasil, 2008-2010).

\begin{tabular}{|c|c|c|c|c|c|c|c|c|c|c|c|c|}
\hline \multirow{4}{*}{$\begin{array}{c}\text { Tipo de } \\
\text { bebida alcoólica } \\
\text { (quintil) }\end{array}$} & \multicolumn{5}{|c|}{ Circunferência da cintura } & \multicolumn{7}{|c|}{ Relação cintura-quadril } \\
\hline & \multirow{2}{*}{\multicolumn{2}{|c|}{$\begin{array}{c}\text { Masculino } \\
\text { Elevada } \\
\end{array}$}} & & \multirow{2}{*}{\multicolumn{2}{|c|}{$\begin{array}{c}\text { Feminino } \\
\text { Elevada }\end{array}$}} & & \multirow{2}{*}{\multicolumn{2}{|c|}{$\begin{array}{c}\text { Masculino } \\
\text { Elevada }\end{array}$}} & \multirow{2}{*}{\multicolumn{4}{|c|}{$\begin{array}{c}\text { Feminino } \\
\text { Elevada } \\
\end{array}$}} \\
\hline & & & & & & & & & & & & \\
\hline & $\mathbf{n}$ & $\%$ & $\begin{array}{l}\text { Valor } \\
\text { de } p^{\star}\end{array}$ & $\mathbf{n}$ & $\%$ & $\begin{array}{l}\text { Valor } \\
\text { de } p^{\star}\end{array}$ & $\mathbf{n}$ & $\%$ & $\begin{array}{l}\text { Valor } \\
\text { de } p^{\star}\end{array}$ & $\mathbf{n}$ & $\%$ & $\begin{array}{l}\text { Valor } \\
\text { de } p^{\star}\end{array}$ \\
\hline Vinho & & & 0,002 & & & 0,132 & & & 0,004 & & & 0,516 \\
\hline $1^{\circ}$ & 240 & 20,3 & & 121 & 34,4 & & 110 & 20,5 & & 216 & 30,9 & \\
\hline $2^{\circ}$ & 269 & 22,7 & & 90 & 25,6 & & 104 & 19,4 & & 177 & 25,3 & \\
\hline $3^{\circ}$ & 172 & 18,2 & & 50 & 14,2 & & 73 & 13,6 & & 91 & 13,0 & \\
\hline $4^{\circ}$ & 240 & 14,5 & & 42 & 11,9 & & 126 & 23,4 & & 108 & 15,5 & \\
\hline $5^{\circ}$ & 261 & 22,0 & & 49 & 13,9 & & 124 & 23,1 & & 107 & 15,3 & \\
\hline Cerveja & & & $<0,001$ & & & $<0,001$ & & & 0,001 & & & $<0,001$ \\
\hline $1^{\circ}$ & 221 & 11,1 & & 167 & 29,5 & & 106 & 11,9 & & 279 & 26,6 & \\
\hline $2^{\circ}$ & 324 & 16,3 & & 121 & 21,4 & & 140 & 15,7 & & 235 & 22,4 & \\
\hline $3^{\circ}$ & 420 & 21,2 & & 112 & 19,8 & & 177 & 19,9 & & 239 & 22,8 & \\
\hline $4^{\circ}$ & 453 & 22,8 & & 98 & 17,3 & & 186 & 21,0 & & 183 & 17,4 & \\
\hline $5^{\circ}$ & 569 & 28,6 & & 68 & 12,0 & & 280 & 31,5 & & 113 & 10,8 & \\
\hline Bebidas destiladas & & & 0,090 & & & 0,887 & & & 0,090 & & & 0,226 \\
\hline $1^{o}$ & 263 & 31,7 & & 46 & 56,8 & & 115 & 29,0 & & 96 & 54,5 & \\
\hline $2^{\circ}$ & 174 & 21,0 & & 16 & 19,7 & & 78 & 19,6 & & 34 & 19,3 & \\
\hline $3^{\circ}$ & 95 & 11,4 & & 10 & 12,4 & & 46 & 11,6 & & 20 & 11,4 & \\
\hline $4^{\circ}$ & 135 & 16,2 & & 5 & 6,2 & & 64 & 16,1 & & 13 & 7,4 & \\
\hline $5^{\circ}$ & 163 & 19,6 & & 4 & 4,9 & & 94 & 23,7 & & 13 & 7,4 & \\
\hline Todas as bebidas & & & $<0,001$ & & & 0,004 & & & $<0,001$ & & & $<0,001$ \\
\hline $1^{\circ}$ & 233 & 10,0 & & 251 & 33,5 & & 118 & 11,0 & & 439 & 30,1 & \\
\hline $2^{o}$ & 327 & 14,0 & & 169 & 22,5 & & 141 & 13,2 & & 320 & 22,5 & \\
\hline $3^{\circ}$ & 458 & 19,5 & & 142 & 18,9 & & 199 & 18,7 & & 309 & 21,7 & \\
\hline $4^{o}$ & 567 & 24,2 & & 111 & 14,8 & & 238 & 22,3 & & 213 & 15,0 & \\
\hline $5^{\circ}$ & 756 & 32,3 & & 77 & 10,3 & & 371 & 34,8 & & 140 & 9,9 & \\
\hline
\end{tabular}

*Teste qui-quadrado.

Fonte: Autores.

senta proteção para o aumento de CC e RCQ e excesso de peso ${ }^{35,36,37}$, neste estudo não verificamos essa associação, pois não foi feita a comparação entre os não bebedores e os quintis de consumo de bebidas alcoólicas. Estudo caso-controle realizado nos Estados Unidos evidenciou menores porcentagens de adiposidade abdominal entre os que consumiam bebidas alcoólicas, quando comparados aos que não consomem, e nas mulheres a adiposidade abdominal tendeu a diminuir com o aumento do consumo de bebidas. $\mathrm{O}$ estudo identificou que, além da quantidade de álcool consumido, o padrão de consumo pode afetar a distribuição de gordura corporal ${ }^{38}$.

Estudo britânico com 7.735 homens de 40 a 59 anos observou que o consumo de bebidas alcoólicas foi associado positivamente às médias de IMC, CC e RCQ. Os resultados mostraram efeitos maiores sobre RCQ e CC do que sobre o IMC. Apesar disso, as variáveis não foram significantes para os tipos de bebidas separadamente ${ }^{11}$. É importante mencionar que o alto consumo de bebidas alcoólicas aumenta o risco para exceder o consumo recomendado de energia diário. A longo prazo, a ingestão diária de energia que excede o gasto energético diário é a força motriz para o ganho de peso. O álcool pode representar de 5\% a $10 \%$ da energia ingerida por adultos, sendo um componente significativo da dieta ${ }^{39}$.

É importante ressaltar que não há consenso sobre o efeito do consumo de bebidas alcoólicas e alteração de parâmetros antropométricos, podendo mostrar tanto associações positivas como negativas e nulas ${ }^{31}$. No presente estudo, encon- 
Tabela 3. Razão de prevalência e intervalos de confiança (IC 95\%) para associação entre consumo de bebidas alcoólicas e circunferência da cintura elevada (ELSA - Brasil, 2008-2010).

\begin{tabular}{|c|c|c|c|c|}
\hline \multirow{2}{*}{ Quintis de consumo } & \multicolumn{2}{|c|}{ Homem } & \multicolumn{2}{|c|}{ Mulher } \\
\hline & Modelo bruto & Modelo ajustado $^{a}$ & Modelo bruto & Modelo ajustado $^{a}$ \\
\hline \multicolumn{5}{|l|}{ Vinho } \\
\hline $1^{\circ}$ quintil & 1 & 1 & 1 & 1 \\
\hline $2^{\circ}$ quintil & $1,04(1,00-1,09)$ & $1,00(0,97-1,03)$ & $0,97(0,93-1,01)$ & $0,99(0,96-1,02)$ \\
\hline $3^{\circ}$ quintil & $1,06(1,01-1,10)$ & $1,02(0,99-1,06)$ & $0,98(0,93-1,04)$ & $0,99(0,95-1,020$ \\
\hline $4^{\mathrm{o}}$ quintil & $1,06(1,02-1,11)$ & $1,00(0,97-1,03)$ & $0,93(0,88-0,98)$ & $0,97(0,94-1,00)$ \\
\hline $5^{\circ}$ quintil & $1,08(1,03-1,12)$ & $1,02(0,99-1,05)$ & $0,98(0,92-1,03)$ & $0,99(0,95-1,03)$ \\
\hline \multicolumn{5}{|l|}{ Cerveja } \\
\hline $1^{\mathrm{o}}$ quintil & 1 & 1 & 1 & 1 \\
\hline $2^{\circ}$ quintil & $1,05(1,01-1,09)$ & $1,01(0,98-1,04)$ & $0,97(0,93-1,01)$ & $0,99(0,96-1,01)$ \\
\hline $3^{\circ}$ quintil & $1,07(1,03-1,11)$ & $1,03(1,00-1,06)$ & $0,98(0,94-1,02)$ & $0,99(0,96-1,02)$ \\
\hline $4^{\circ}$ quintil & $1,08(1,04-1,12)$ & $1,04(1,01-1,07)$ & $1,03(0,98-1,08)$ & $1,02(0,98-105)$ \\
\hline $5^{\circ}$ quintil & $1,10(1,06-1,14)$ & $1,05(1,02-1,08)$ & $1,10(1,04-1,17)$ & $1,03(1,00-1,07)$ \\
\hline \multicolumn{5}{|l|}{ Bebidas destiladas } \\
\hline $1^{\circ}$ quintil & 1 & 1 & 1 & 1 \\
\hline $2^{\circ}$ quintil & $0,99(0,94-1,03)$ & $0,99(0,96-1,02)$ & $0,98(0,90-1,07)$ & $1,00(0,94-1,07)$ \\
\hline $3^{\circ}$ quintil & $1,05(1,00-1,11)$ & $1,05(1,01-1,10)$ & $1,05(0,93-1,19)$ & $1,01(0,93-1,11)$ \\
\hline $4^{\circ}$ quintil & $1,04(0,99-1,09)$ & $0,99(0,96-1,03)$ & $1,01(0,87-1,17)$ & $1,00(0,90-1,11)$ \\
\hline $5^{\circ}$ quintil & $0,99(0,94-1,04)$ & $1,00(0,96-1,03)$ & $0,97(0,84-1,12)$ & $0,98(0,90-1,08)$ \\
\hline \multicolumn{5}{|l|}{ Todas as bebidas } \\
\hline $1^{\circ}$ quintil & 1 & 1 & 1 & 1 \\
\hline $2^{\circ}$ quintil & $1,04(1,00-1,08)$ & $1,02(0,99-1,05)$ & $0,97(0,94-1.00)$ & $0,97(0,94-0,99)$ \\
\hline $3^{\circ}$ quintil & $1,07(1,03-1,11)$ & $1,03(1,01-1,06)$ & $0,97(0,94-1,01)$ & $0,98(0,96-1,01)$ \\
\hline $4^{\circ}$ quintil & $1,09(1,05-1,13)$ & $1,04(1,02-1,07)$ & $0,99(0,95-1,03)$ & $0,99(0,97-1,02)$ \\
\hline $5^{\circ}$ quintil & $1,12(1,09-1,16)$ & $1,05(1,03-1,08)$ & $1,07(1,01-1,12)$ & $1,02(0,99-1,06)$ \\
\hline
\end{tabular}

${ }^{a}$ Modelo ajustado para idade, índice de massa corporal, renda per capita, atividade física e tabagismo.

Fonte: Autores.

trou-se associação entre CC e RCQ elevadas com o consumo de cerveja, dado que pode ser explicado pelo consumo predominante de cerveja entre os brasileiros ${ }^{25}$. Além disso, como a cerveja é consumida em quantidade mais elevada, fornece maior aporte energético quando comparado a outros tipos de bebidas alcoólicas, o que pode influenciar em um maior acúmulo de gordura.

Nesse contexto, o padrão de beber e comer é crucial, ou seja, se o álcool é adicionado a fontes de alimentos habituais ou se ele é substituído por energia de alimentação habitual tem um impacto diferente. Além disso, a composição do alimento ao qual é adicionado é de grande relevância. Não é possível a avaliação correta desse comportamento de consumo em estudos epidemiológicos. A quantidade absoluta e a frequência do consumo de bebidas alcoólicas determinam a via metabólica (via álcool desidrogenase - ADH - ou via microssomal de oxidação de etanol - MEOS) de degradação do álcool, e assim as interações com o metabolismo energético, bem como com outro sistema fisiológico ${ }^{15,40,41}$. A via $\mathrm{ADH}$ é responsável por maior liberação de energia e ocorre em bebedores sociais, e a MEOS é utilizada com maior frequência em bebedores excessivos. O fígado é determinante para o metabolismo tanto de lipídios quanto do álcool, uma vez que a metabolização dos lipídios não é realizada, para dar preferência à do álcool, ocorrendo um balanço energético positivo de gorduras e sua consequente acumulação, ou seja, ganho de peso ${ }^{15}$.

O estudo apresenta algumas limitações, entre as quais está o delineamento transversal. Como os participantes não foram acompanhados, não se pode estabelecer relação causa e efeito entre os desfechos e o consumo de bebida alcóolica. Outra limitação é o instrumento de coleta de dados, que por ser um questionário de autorrelato pode superestimar ou subestimar o consumo de bebidas 
Tabela 4. Razão de prevalência e intervalos de confiança (IC 95\%) para associação entre consumo de bebidas alcoólicas e relação cintura-quadril elevada (ELSA - Brasil, 2008-2010).

\begin{tabular}{|c|c|c|c|c|}
\hline \multirow{2}{*}{ Quintis de consumo } & \multicolumn{2}{|c|}{ Homem } & \multicolumn{2}{|c|}{ Mulher } \\
\hline & Modelo bruto & Modelo ajustado $^{a}$ & Modelo bruto & Modelo ajustado $^{a}$ \\
\hline \multicolumn{5}{|l|}{ Vinho } \\
\hline $1^{\circ}$ quintil & 1 & 1 & 1 & 1 \\
\hline $2^{\circ}$ quintil & $0,99(0,95-1,03)$ & $0,96(0,93-1,00)$ & $0,98(0,94-1,02)$ & $0,98(0,95-1,02)$ \\
\hline $3^{\circ}$ quintil & $1,01(0,97-1,07)$ & $0,98(0,94-1,02)$ & $0,98(0,93-1,04)$ & $0,98(0,93-1,03)$ \\
\hline $4^{\circ}$ quintil & $1,07(1,02-1,12)$ & $1,01(0,97-1,06)$ & $0,98(0,93-1,04)$ & $1,01(0,96-1,06)$ \\
\hline $5^{\circ}$ quintil & $1,05(1,00-1,10)$ & $1,00(0,96-1,04)$ & $1,03(0,97-1,08)$ & $1,03(0,97-1,08)$ \\
\hline \multicolumn{5}{|l|}{ Cerveja } \\
\hline $1^{\circ}$ quintil & 1 & 1 & 1 & 1 \\
\hline $2^{\circ}$ quintil & $1,00(0,96-1,05)$ & $0,99(0,95-1,02)$ & $1,01(0,97-1,05)$ & $1,01(0,97-1,05)$ \\
\hline $3^{\circ}$ quintil & $1,01(0,97-1,05)$ & $1,00(0,96-1,03)$ & $1,05(0,01-1,10)$ & $1,05(1,01-1,09)$ \\
\hline $4^{\circ}$ quintil & $1,01(0,97-1,05)$ & $0,99(0,96-1,03)$ & $1,09(1,04-1,14)$ & $1,07(1,03-1,12)$ \\
\hline $5^{\circ}$ quintil & $1,06(1,02-1,10)$ & $1,03(1,00-1,07)$ & $1,15(1,09-1,21)$ & $1,10(1,04-1,15)$ \\
\hline \multicolumn{5}{|l|}{ Bebidas destiladas } \\
\hline $1^{\circ}$ quintil & 1 & 1 & 1 & 1 \\
\hline $2^{\circ}$ quintil & $1,00(0,95-1,05)$ & $0,99(0,95-1,03)$ & $0,98(0,89-1,07)$ & $0,98(0,90-1,06)$ \\
\hline $3^{\circ}$ quintil & $1,05(0,98-1,13)$ & $1,03(0,97-1,09)$ & $1,07(0,96-1,20)$ & $1,04(0,94-1,16)$ \\
\hline $4^{\circ}$ quintil & $1,04(0,98-1,10)$ & $0,98(0,93-1,03)$ & $1,10(0,96-1,26)$ & $1,04(0,90-1,20)$ \\
\hline $5^{\circ}$ quintil & $1,05(1,00-1,11)$ & $1,02(0,98-1,07)$ & $1,10(0,96-1,26)$ & $1,12(1,00-1,27)$ \\
\hline \multicolumn{5}{|l|}{ Todas as bebidas } \\
\hline $1^{\circ}$ quintil & 1 & 1 & 1 & 1 \\
\hline $2^{\circ}$ quintil & $0,99(0,95-1,03)$ & $0,99(0,96-1,02)$ & $0,98(0,94-1,01)$ & $0,98(0,95-1,01)$ \\
\hline $3^{\circ}$ quintil & $1,01(0,97-1,05)$ & $0,98(0,95-1,02)$ & $1,03(1,00-1,07)$ & $1,03(1,00-1,06)$ \\
\hline $4^{\circ}$ quintil & $1,01(0,97-1,05)$ & $0,98(0,95-1,01)$ & $1,02(0,98-1,06)$ & $1,01(0,98-1,05)$ \\
\hline $5^{\circ}$ quintil & $1,06(1,03-1,10)$ & $1,01(0,98-1,05)$ & $1,12(1,07-1,17)$ & $1,07(1,03-1,12)$ \\
\hline
\end{tabular}

${ }^{a}$ Modelo ajustado para idade, índice de massa corporal, renda per capita, atividade física e tabagismo.

Fonte: Autores.

alcoólicas, como o consumo de destiladas, que pode ser omitido por se saber o efeito maléfico à saúde, além de ter seu consumo estigmatizado por ser mais consumido por alcoolistas crônicos. Entretanto, é um tipo de coleta de dados muito utilizado em grandes estudos epidemiológicos.

Como a obesidade tem determinação multifatorial, é de fato complexa a avaliação da influência independente do consumo de bebidas alcoólicas no risco de adiposidade abdominal. Os estudos epidemiológicos são importantes para mostrar um efeito desse consumo sobre a vulnerabilidade do aumento da CC e da RCQ. Para estudos futuros acerca dessa associação, é importante avaliar a relação da dieta dos indivíduos e o consumo de bebidas alcoólicas. Apesar de se saber que a contribuição energética do consumo de bebidas alcoólicas é considerável na dieta, é im- portante compreender a contribuição de outros alimentos na gênese da adiposidade abdominal.

\section{Conclusão}

Foi encontrada associação entre o consumo de cerveja e a inadequação da CC e da RCQ em homens e mulheres. É possível identificar a relevância dos resultados deste estudo para melhor compreensão do impacto do consumo de bebida alcóolica no âmbito da saúde pública, já que o álcool é um importante fator de risco para a saúde e seu consumo vem aumentando, em especial entre os mais jovens. Esse fato pode contribuir bastante para o aumento precoce do risco cardiovascular, de forma que a diminuição de seu consumo constitui um desafio para as políticas públicas de saúde. 


\section{Colaboradores}

GG Torres trabalhou na concepção, delineamento, análise e interpretação dos dados, na redação do artigo e sua revisão crítica. JH Siqueira, OG Enriquez e TS Pereira contribuíram na concepção, delineamento, análise e interpretação dos dados, na redação do artigo, sua revisão crítica e aprovação da versão a ser publicada. GV Melendez, BB Duncan e AC Goulart atuaram na concepção, delineamento, análise e interpretação dos dados e na aprovação da versão a ser publicada. MCB Molina auxiliou na concepção, delineamento, aquisição de dados, revisão crítica do conteúdo e aprovação final de sua versão para publicação.

\section{Referências}

1. Abarca-Gómez L, Abdeen ZA, Hamid ZA, Abu-Rmeileh NM, Acosta-Cazares B, Acuin C, NCD Risk Factor Collaboration (NCD-RisC). Worldwide trends in body-mass index, underweight, overweight, and obesity from 1975 to 2016: a pooled analysis of 2416 population-based measurement studies in 128.9 million children, adolescents, and adults. Lancet 2017; 390(10113):2627-2642.

2. Romdhani A. Apovian CM. Obesity: definition, comorbidities, causes, and burden. Am J Manag Care 2016; 22(7 Suppl.):176-185.

3. Després JP, Moorjani S, Lupien PJ, Tremblay A, Nadeau A, Bouchard C. Regional distribution of body fat, plasma lipoproteins, and cardiovascular disease. Arterioscler An Off J Am Hear Assoc Inc 1990; 10(4):497511.

4. Choi D, Choi S, Son JS, Oh SW, Park SM. Impact of discrepancies in general and abdominal obesity on major adverse cardiac events. J Am Heart Assoc 2019; 8(18):e013471.

5. Bennasar-Veny M, Lopez-Gonzalez AA, Tauler P, Cespedes ML, Vicente-Herrero T, Yañez A, Tomas-Salva $\mathrm{M}$, Aguilo A. Body adiposity index and cardiovascular health risk factors in Caucasians: a comparison with the body mass index and others. PLoS One 2013; 8(5):e63999.

6. Mitsuhashi K, Hashimoto Y, Tanaka M, Toda H, Matsumoto S, Ushigome E, Asano M, Yamazaki M, Oda Y, Fukui M. Combined effect of body mass index and waist-height ratio on incident diabetes; a population based cohort study. J Clin Biochem Nutr 2017; 61(2):118-122.

7. Janssen I, Katzmarzyk PT, Ross R. Waist circumference and not body mass index explains obesity-related health risk. Am J Clin Nutr 2004; 79(3):379-384.

8. Pinho CPS, Diniz ADS, Arruda IKG, Batista Filho M, Coelho PC, Sequeira LADS, Lira PIC. Prevalência e fatores associados à obesidade abdominal em indivíduos na faixa etária de 25 a 59 anos do Estado de Pernambuco, Brasil. Cad Saude Publica 2013; 29(2):313-324.
9. Ferreira APDS, Szwarcwald CL, Damacena GN. Prevalência e fatores associados da obesidade na população brasileira: estudo com dados aferidos da Pesquisa Nacional de Saúde, 2013. Rev Bras Epidemiol 2019; 22: e190024.

10. Wannamethee SG, Shaper AG, Whincup PH. Alcohol and adiposity: effects of quantity and type of drink and time relation with meals. Int J Obes (Lond) 2005; 29(12):1436-1444.

11. Lourenço S, Oliveira A, Lopes C. The effect of current and lifetime alcohol consumption on overall and central obesity. Eur J Clin Nutr 2012; 66(7):813-818.

12. World Health Organization (WHO). Global Status Report on Alcohol and Health 2004. Geneva: WHO; 2004.

13. GBD 2016 Alcohol Collaborators. Alcohol use and burden for 195 countries and territories, 1990-2016: a systematic analysis for the Global Burden of Disease Study 2016. Lancet 2018; 392(10152):1015-1035.

14. Stockwell T, Zhao J, Panwar S, Roemer A, Naimi T, Chikritzhs T. Do "moderate" drinkers have reduced mortality risk? A systematic review and meta-analysis of alcohol consumption and all-cause mortality. J Stud Alcohol Drugs 2016; 77(2):185-198.

15. Suter PM, Hasler E, Vetter W. Effects of alcohol on energy metabolism and body weight regulation: is alcohol a risk factor for obesity? Nutr Rev 2009; 55(5):157-171.

16. Lieber CS. Perspectives: do alcohol calories count? Am J Clin Nutr 1991; 54(6):976-982.

17. Haffner SM, Stern MP, Hazuda HP, Pugh J, Patterson JK, Malina R. Upper body and centralized adiposity in Mexican Americans and non-Hispanic whites: relationship to body mass index and other behavioral and demographic variables. Int J Obes 1986; 10(6):493-502.

18. Kaye SA, Folsom AR, Prineas RJ, Potter JD, Gapstur SM. The association of body fat distribution with lifestyle and reproductive factors in a population study of postmenopausal women. Int J Obes 1990; 14(7):583-591. 
19. Enríquez Martínez OG, Luft VC, Faria CP, Molina MDCB. Alcohol consumption and lipid profile in participants of the Longitudinal Study of Adult Health (ELSA-BRASIL). Nutr Hosp 2019; 36(3):665-673.

20. Duncan BB, Chambless LE, Schmidt MI, Folsom AR, Szklo M, Crouse JR 3rd, et al. Association of the waist-to-hip ratio is different with wine than with beer or hard liquor consumption. Atherosclerosis risk in communities study investigators. Am J Epidemiol 1995; 142(10):1034-1038.

21. Vadstrup ES, Petersen L, Sørensen TIA, Grønbæk M. Waist circumference in relation to history of amount and type of alcohol: results from the Copenhagen City Heart Study. 2003; 27(2):238-246.

22. Schmidt MI, Duncan BB, Mill JG, Lotufo PA, Chor D, Barreto SM, Aquino EM, Passos VM, Matos SM, Molina Mdel C, Carvalho MS, Bensenor IM. Cohort profile: Longitudinal study of adult health (ELSA-Brasil). Int J Epidemiol 2015; 44(1):68-75.

23. Mill JG, Pinto K, Griep RH, Goulart A, Foppa M, Lotufo PA, et al. Aferições e exames clinicos realizados nos participantes do ELSA-Brasil. Rev Saude Publica 2013; 47(Supl. 2):54-62.

24. Lohman TG, Roche AF, Martorell R. Antthropometric standardization reference manual. Champaign: $\mathrm{Hu}-$ man Kinetics Books; 1988.

25. World Health Organization (WHO). Obesity: preventing and managing the global epidemic. Report of a Who Consultation on Obesity. Geneva: WHO; 1998. [cited 2020 Jul 5]. Available from: http://www.ncbi. nlm.nih.gov/pubmed/11234459

26. Chor D, Alves MG, Giatti L, Cade NV, Nunes MA, Molina MDC, Benseñor IM, Aquino EM, Passos V, Santos SM, Fonseca MJ, Oliveira LC. Questionário do ELSA-Brasil: desafios na elaboração de instrumento multidimensional. Rev Saude Publica 2013; 47(Supl. 2):27-36.

27. Matsudo S, Araújo T, Matsudo V, Andrade D, Andrade E, Oliveira LC, Braggion G. Questionário Internacional de Atividade Física (Ipaq): estupo de validade e reprodutibilidade no Brasil. Rev Bras Atividade Física Saúde 2012; 6(2):5-18.

28. Ferreira MG, Valente JG, Gonçalves-Silva RMV, Sichieri R. Alcohol consumption and abdominal fat in blood donors. Rev Saude Publica 2008; 42(6):1067-1073.

29. Machado PAN, Sichieri R. Relação cintura-quadril e fatores de dieta em adultos. Rev Saude Publica 2002; 36(2):198-204.

30. Bendsen NT, Christensen R, Bartels EM, Kok FJ, Sierksma A, Raben A, et al. Is beer consumption related to measures of abdominal and general obesity? A systematic review and meta-analysis.Nutr Rev 2013; 71(2):67-87.

31. Guimarães NS, Nemer ASA, Fausto MA. Influência do consumo de álcool nas alterações antropométricas: uma revisão sistemática. Nutr Clin Diet Hosp 2013; 33(3):68-76.

32. Pajari M, Pietilainen KH, Kaprio J, Rose RJ, Saarni SE. The effect of alcohol consumption on later obesity in early adulthood - a population-based longitudinal study. Alcohol Alcohol 2010; 45(2):173-179.

33. Wolle CC, Sanches M, Zilberman ML, Caetano R, Zaleski M, Laranjeira RR, Pinsky I. Differences in drinking patterns between men and women in Brazil. Rev Bras Psiquiatr 2011; 33(4):367-373.
34. Ng M, Fleming T, Robinson M, Thomson B, Graetz N, Margono C, Mullany EC, Biryukov S, Abbafati C, Abera SF, Abraham JP, Abu-Rmeileh NM, Achoki T, AlBuhairan FS, Alemu ZA, Alfonso R, Ali MK, Ali R, Guzman NA, Ammar W, Anwari P, Banerjee A, Barquera S, Basu S, Bennett DA, Bhutta Z, Blore J, Cabral N, Nonato IC, Chang JC, Chowdhury R, Courville KJ, Criqui MH, Cundiff DK, Dabhadkar KC, Dandona L, Davis A, Dayama A, Dharmaratne SD, Ding EL, Durrani AM, Esteghamati A, Farzadfar F, Fay DF, Feigin VL, Flaxman A, Forouzanfar MH, Goto A, Green MA, Gupta R, Hafezi-Nejad N, Hankey GJ, Harewood HC, Havmoeller R, Hay S, Hernandez L, Husseini A, Idrisov BT, Ikeda N, Islami F, Jahangir E, Jassal SK, Jee SH, Jeffreys M, Jonas JB, Kabagambe EK, Khalifa SE, Kengne AP, Khader YS, Khang YH, Kim D, Kimokoti RW, Kinge JM, Kokubo Y, Kosen S, Kwan G, Lai T, Leinsalu M, Li Y, Liang X, Liu S, Logroscino G, Lotufo PA, Lu Y, Ma J, Mainoo NK, Mensah GA, Merriman TR, Mokdad AH, Moschandreas J, Naghavi M, Naheed A, Nand D, Narayan KM, Nelson EL, Neuhouser ML, Nisar MI, Ohkubo T, Oti SO, Pedroza A, Prabhakaran D, Roy N, Sampson U, Seo H, Sepanlou SG, Shibuya K, Shiri R, Shiue I, Singh GM, Singh JA, Skirbekk V, Stapelberg NJ, Sturua L, Sykes BL, Tobias M, Tran BX, Trasande L, Toyoshima H, van de Vijver S, Vasankari TJ, Veerman JL, Velasquez-Melendez G, Vlassov VV, Vollset SE, Vos T, Wang C, Wang X, Weiderpass E, Werdecker A, Wright JL, Yang YC, Yatsuya H, Yoon J, Yoon SJ, Zhao Y, Zhou M, Zhu S, Lopez AD, Murray CJ, Gakidou E. Global, regional, and national prevalence of overweight and obesity in children and adults during 19802013: a systematic analysis for the Global Burden of Disease Study 2013. Lancet 2014; 384(9945):766-781.

35. Wakabayashi I. Relationship between alcohol intake and lipid accumulation product in middle-aged men. Alcohol Alcohol 2013; 48(5):535-542.

36. Janssens JP, Bruckers L, Joossens J V., Molenberghs G, Vinck J, Renard D, Tafforeau J. Overweight, obesity and beer consumption. Alcohol drinking habits in Belgium and body mass index. Arch Public Heal 2001; 59(5-6):223-238

37. Traversy G, Chaput J-P. Alcohol consumption and obesity: an update. Curr Obes Rep 2015; 4(1):122-130.

38. Dorn JM, Hovey K, Muti P, Freudenheim JL, Russell M, Nochajski TH, Trevisan M. Alcohol drinking patterns differentially affect central adiposity as measured by abdominal height in women and men. J Nutr 2003; 133(8):2655-2662.

39. Schröder H, Morales-Molina JA, Bermejo S, Barral D, Mándoli ES, Grau M, Guxens M, de Jaime Gil E, Alvarez MD, Marrugat J. Relationship of abdominal obesity with alcohol consumption at population scale. Eur J Nutr 2007; 46(7):369-376.

40. Frezza M, Di Padova C, Pozzato G, Terpin M, Baraona E, Lieber CS. High blood alcohol levels in women. $N$ Engl J Med 1990; 322(2):95-99.

41. Vannucchi H, Marchini JS. Nutrição Clínica. In: Vannucchi H. Alcoolismo e nutrição. Rio de Janeiro: Guanabara Koogan; 2007. p. 137-155.

Artigo apresentado em 17/08/2020

Aprovado em 18/02/2021

Versão final apresentada em 20/02/2021

Editores-chefes: Romeu Gomes, Antônio Augusto Moura da Silva 